\title{
Case report of dobutamine stress echocardiography selecting treatment strategy after acute pulmonary edema in a patient with acute coronary syndrome and severly depressed left ventricular function
}

\section{DMarko Hranilović*, DBranko Ostrički, DMihajlo Kovačić}

Čakovec County Hospital, Čakovec, Croatia

\author{
KEYWORDS: dobutamine stress echocardiography, viability assessment, myocardial revasculariza- \\ tion, acute coronary syndrome, pulmonary edema. \\ CITATION: Cardiol Croat. 2019;14(3-4):107-8. | https://doi.org/10.15836/ccar2019.107
}

*ADDRESS FOR CORRESPONDENCE: Marko Hranilović, Županijska bolnica Čakovec, I.G.Kovačića 1E, HR-40000 Čakovec, Croatia. / Phone: +385-95-9030297 / E-mail: markohranilovic@yahoo.com

ORCID: Marko Hranilović, https://orcid.org/0000-0001-6522-5882 • Branko 0strički, https://orcid.org/0000-0001-8682-5158 Mihajlo Kovačić, https://orcid.org/0000-0002-2577-9474

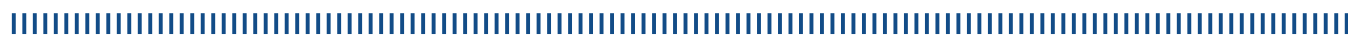

Introduction: Assessment of myocardial viability can be done with a variety of imaging techniques, including nuclear, magnetic resonance and echocardiographic methods. Stress echocardiography offers higher specificity for postoperative ejection fraction improvement than perfusion techniques due to the requirement of viable myocardium with preserved contractile reserve. ${ }^{1,2}$ Low dose dobutamine is the preferred method for viability assessment. Myocardial revascularization without detected viability may be considered according to guidelines but portends worse prognosis ${ }^{3}$ and in this case, if feasible, left ventricular assist devices and heart transplantation may be considered. Dipyridamole in very low doses can be used for viability detection and may be considered due to a better safety profile than dobutamine in patients with uncontrolled hypertension or tachyarrythmias ${ }^{4}$

Case report: 64-years-old male patient, formerly treated for hypertension, type 2 diabetes mellitus and peripheral vascular disease (conservative therapy) was admitted to coronary care unit due to chest pain with modest troponin elevation. Coronary angiography was urgently performed and revealed highly significant stenoses in proximal parts of all coronary vessels with high syntax score (Figure $\mathbf{1}$, Figure 2). Immediate referral to the cardiac surgeon was suggested by an interventional cardiologist
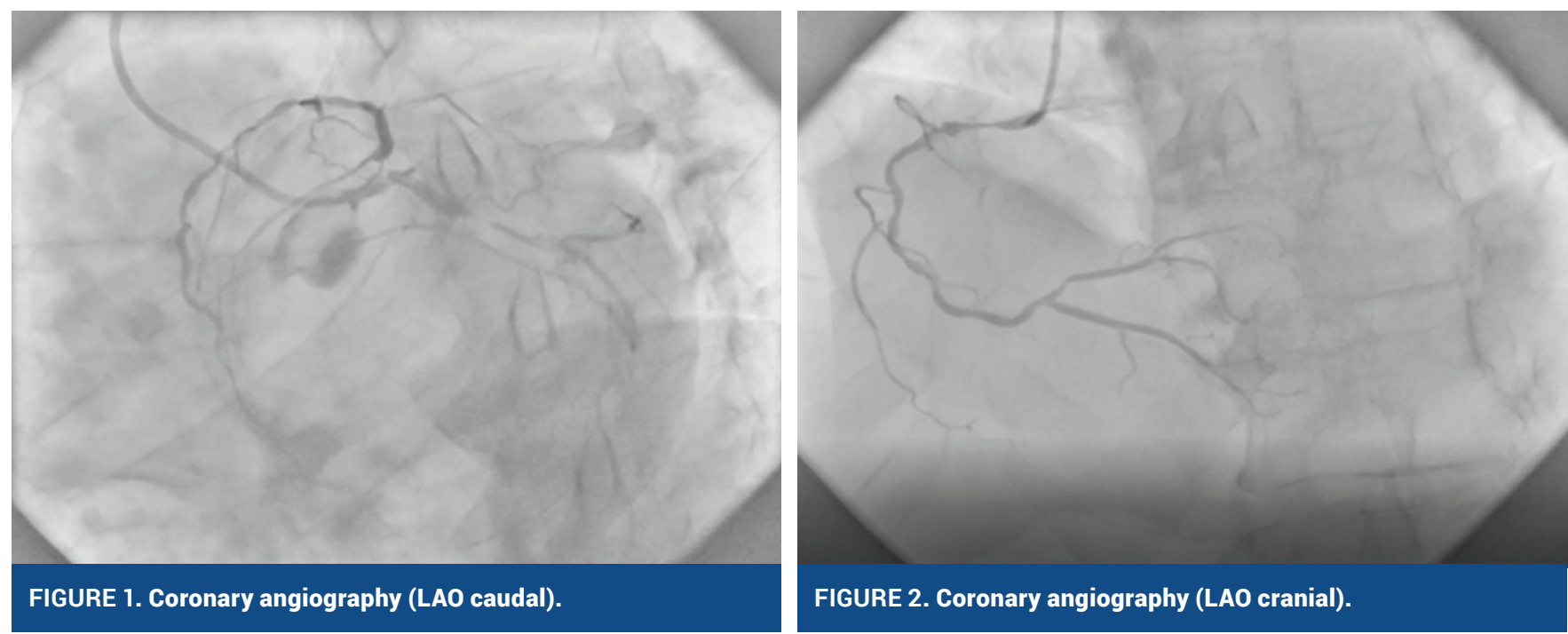

RECEIVED:

February 28, 2019

ACCEPTED:

March 24, 2019

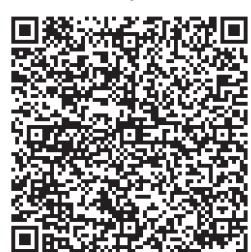

but upon completion of diagnostic procedure pulmonary edema was induced due to contrast infusion and hypertensive reaction (RR 180/100). Despite urgent treatment with parenteral nitrate, loop diuretics, morphine and non-invasive ventilation significant lactacidosis with $\mathrm{pH} 7.1$ and acute respiratory failure ensued $\left(\mathrm{SO}_{2} 72 \%\right.$ ). Bedside echocardiography showed left ventricular ejection fraction (LVEF) $30 \%$ with hypo/akinesia in all vascular territories. Patient was sedated, intubated and mechanically ventilated for a few hours. When he was clinically stabilized heart team opted for viability assessment in order to plan treatment (in case of no viability detected left ventricular assist device as a bridge to heart transplantation would be an option). Serial troponins showed only modest elevation above reference values. We opted for a low dose dobutamine protocol (up to $10 \mathrm{mcg} / \mathrm{kg} / \mathrm{min}$ ) when the patient 
Case report of dobutamine stress echocardiography selecting treatment strategy after acute pulmonary edema in a patient with acute coronary syndrome and severly depressed left ventricular function

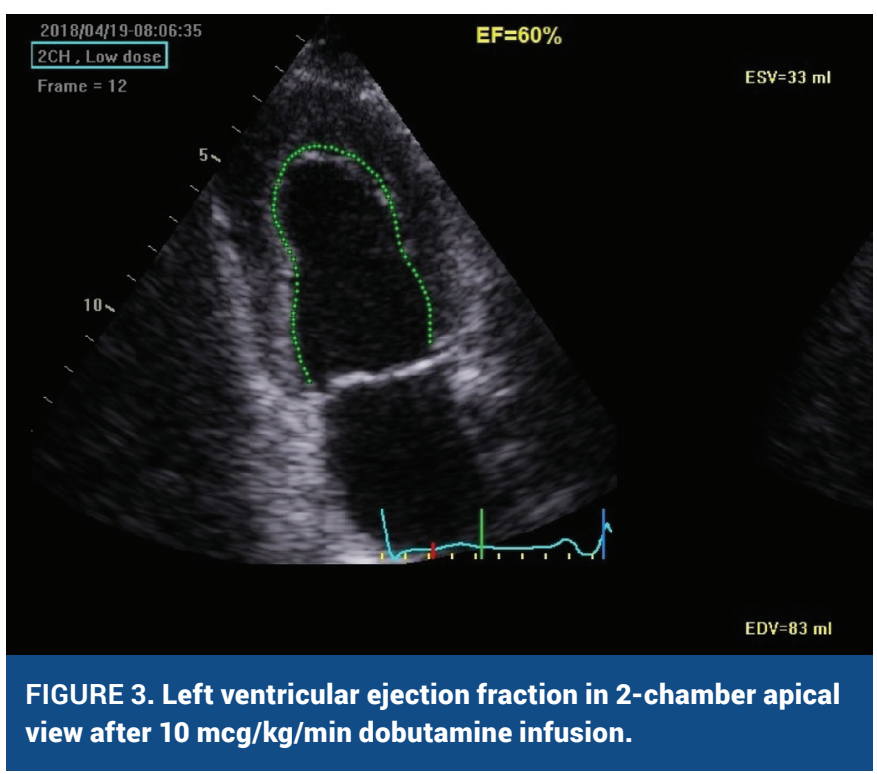

was normotensive and clinically stable and verified LVEF increase to 54\% (by Simpson method measuring 2- and 4-chamber with auto LVEF by speckle tracking) (Figure 3). The patient was transferred to cardiac surgery and had received LIMA to LAD and RIMA to PD which was a part of the right dominant coronary artery. Subsequently we documented good functional recovery (stress ECG test 4 months later was stopped after 6 minutes of Bruce protocol due to peripheral claudication with no chest pain or dyspnea and without significant ECG changes). Echocardiography 10 months after revascularization showed LVEF of 55\% with normokynesia in all segments of myocardium. MSCT angiography verified bilateral femoral artery occlusion. With walking distance of 500 meters he has not been scheduled for operative/interventional treatment at this point in time and has completed hospital cardiac rehabilitation programme.

Conclusion: Different imaging modalities can be used for viability detection in severely depressed left ventricular function. Pharmacological stress imaging has higher specificity for improvement of ejection fraction after revascularization than perfusion-based tests and may be done if the risk is perceived acceptable as it was in our patient after clinical stabilization.

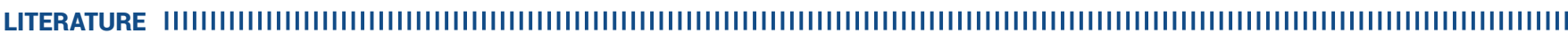

1. Barilla F, Gheorghiade M, Alam M, Khaja F, Goldstein S. Low-dose dobutamine in patients with acute myocardial infarction identifies viable but not contractile myocardium and predicts the magnitude of improvement in wall motion abnormalities in response to coronary revascularization. Am Heart J. 1991 Dec;122(6):1522-31. https://doi.org/10.1016/0002-8703(91)90267-L

2. Bax JJ, Wijns W, Cornel JH, Visser FC, Boersma E, Fioretti PM. Accuracy of currently available techniques for prediction of functional recovery after revascularization in patients with left ventricular dysfunction due to chronic coronary artery disease: comparison of pooled data. J Am Coll Cardiol. 1997 Nov 15;30(6):1451-60. https://doi.org/10.1016/S0735-1097(97)00352-5

3. Meluzín J, Cerný J, Frélich M, Stetka F, Spinarová L, Popelová J, et al. Prognostic value of the amount of dysfunctional but viable myocardium in revascularized patients with coronary artery disease and left ventricular dysfunction. Investigators of this Multicenter Study. J Am Coll Cardiol. 1998 0ct;32(4):912-20. https://doi.org/10.1016/S0735-1097(98)00324-6

4. Sicari R, Ripoli A, Picano E, Borges AC, Varga A, Mathias W, et al; VIDA (Viability Identification with Dipyridamole Administration) Study Group. The prognostic value of myocardial viability recognized by low dose dipyridamole echocardiography in patients with chronic ischaemic left ventricular dysfunction. Eur Heart J. 2001 May;22(10):837-44. https://doi.org/10.1053/euhj.2000.2322 